Check for updates

Cite this: Chem. Commun., 2018, 54, 13022

Received 17th October 2018, Accepted 31st October 2018

DOI: $10.1039 / \mathrm{c} 8 \mathrm{cc} 08328 \mathrm{~b}$

rsc.li/chemcomm

\section{Nitrosonium ion catalysis: aerobic, metal-free cross-dehydrogenative carbon-heteroatom bond formation $\dagger$}

\author{
Luis Bering, (D) ab Laura D'Ottavio, ${ }^{\text {ac }}$ Giedre Sirvinskaite ${ }^{a}$ and \\ Andrey P. Antonchick (D)*ab
}

\begin{abstract}
Catalytic cross-dehydrogenative coupling of heteroarenes with thiophenols and phenothiazines has been developed under mild and environmentally benign reaction conditions. For the first time, $\mathrm{NO}_{x}{ }^{+}$was applied for catalytic $\mathrm{C}-\mathrm{S}$ and $\mathrm{C}-\mathrm{N}$ bond formation. A comprehensive scope for the $\mathrm{C}-\mathrm{H} / \mathrm{S}-\mathrm{H}$ and $\mathrm{C}-\mathrm{H} / \mathrm{N}-\mathrm{H}$ crossdehydrogenative coupling was demonstrated with $>60$ examples. The sustainable cross-coupling conditions utilize ambient oxygen as the terminal oxidant, while water is the sole by-product.
\end{abstract}

The formation of carbon-heteroatom bonds is fundamental for the synthesis of natural products, pharmaceuticals and materials science. $^{1}$ To overcome the requirement for pre-functionalized starting materials, cross-dehydrogenative coupling (CDC) has emerged as a highly efficient strategy. ${ }^{2}$ Transition-metal-catalyzed $\mathrm{C}-\mathrm{S}$ and $\mathrm{C}-\mathrm{N}$ bond formation has been widely reported. ${ }^{3}$ Cost, toxicity and oxygen sensitivity of catalysts limit the general applicability. ${ }^{4}$ Consequently, metal-free synthesis has gained increasing interest. ${ }^{5}$ Different metal-free approaches for the $\mathrm{C}-\mathrm{H} / \mathrm{S}-\mathrm{H}$ CDC have been reported. ${ }^{6}$ Additionally, the unique dehydrogenative amination with phenothiazines has received significant attention. ${ }^{7}$ High temperatures, excess of oxidants and harmful solvents are common limitations.

Nitronium and nitrosonium salts are inexpensive, stable and non-toxic single-electron oxidants. ${ }^{8}$ Radner's group reported the synthesis of biaryls using $\mathrm{NOBF}_{4}$ as catalyst (Fig. 1a). ${ }^{9}$ Ambient oxygen was identified as the terminal oxidant and water as the by-product. ${ }^{10}$ Later, Wang's group reported the catalytic intramolecular $\mathrm{C}-\mathrm{C}$ bond formation (Fig. 1b) $\cdot{ }^{11}$ Under acidic reaction conditions, $\mathrm{NO}^{+}$is generated in situ from $\mathrm{NaNO}_{2}$. The oxidative coupling of phenols is well studied. ${ }^{12}$ Recently, our group

\footnotetext{
${ }^{a}$ Department of Chemical Biology, Max-Planck-Institute of Molecular Physiology, Otto-Hahn-Straße 11, 44227 Dortmund, Germany.

E-mail: Andrey.Antonchick@mpi-dortmund.mpg.de

${ }^{b}$ Faculty of Chemistry and Chemical Biology, TU Dortmund University,

Otto-Hahn-Straße 4a, 44227 Dortmund, Germany

${ }^{c}$ University of Bologna, Department of Pharmacy and Biotechnology,

Via Belmeloro 6, 40126 Bologna, Italy

† Electronic supplementary information (ESI) available. See DOI: 10.1039/c8cc08328b
}

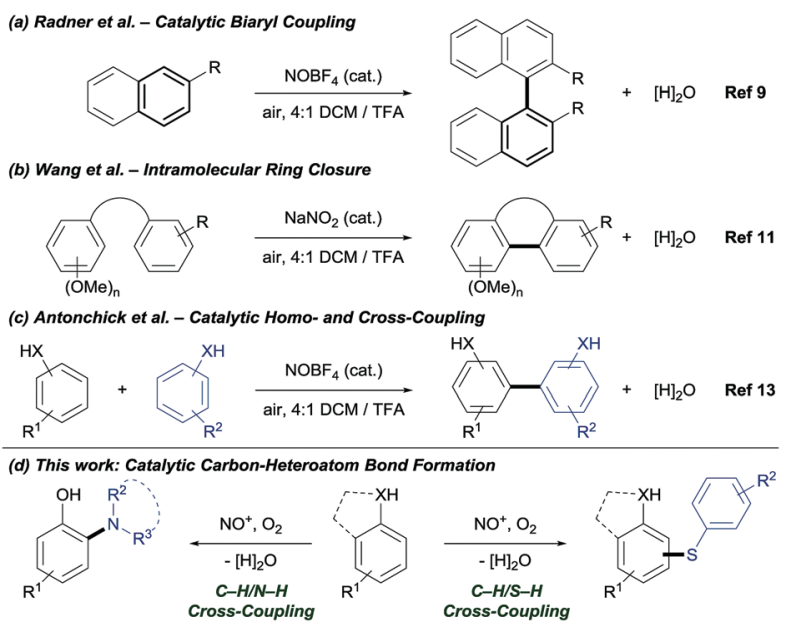

Fig. 1 Prior work on the oxidative carbon-carbon bond formation via $\mathrm{C}-\mathrm{H}$ bond functionalization and newly developed transformation catalyzed by $\mathrm{NO}_{x}^{+}$.

reported the $\mathrm{NO}^{+}$catalyzed coupling for the construction of $\mathrm{C}-\mathrm{C}$ bonds. ${ }^{13}$ Despite the impact of $\mathrm{NO}^{+}$as catalyst for oxidative C-C bond formation, the application in carbon-heteroatom bond formation via $\mathrm{C}-\mathrm{H}$ bond functionalization is unprecedented. Herein, we demonstrate the first $\mathrm{NO}_{x}^{+}$catalyzed C-H/S-H and $\mathrm{C}-\mathrm{H} / \mathrm{N}-\mathrm{H}$ CDC under mild and environmentally benign reaction conditions (Fig. 1d).

Nitrosonium salts are capable to convert thiols to disulfides. ${ }^{14}$ Oxidation of thiols proceeds via transient $S$-nitrosation and recombination of $S$-centred radicals. Due to the low bond dissociation energy (BDE) of phenols and thiophenols, the possibility for a radical-radical recombination reaction of phenoxy and sulfur radicals was hypothesized. ${ }^{15} \mathrm{~A}$ multi parameter optimization for the cross-coupling of $p$-cresol (1a) and 4-chlorothiophenol (2a) was performed (Table S1, ESI $\dagger$ ). To our delight, 3a was isolated in excellent yield, by using $\mathrm{NO}_{2} \mathrm{BF}_{4}$ as the catalyst. Hexafluoroisopropanol (HFIP) was identified as the best solvent, due to its acidic character and the unique ability to stabilize radical intermediates. ${ }^{16}$ 


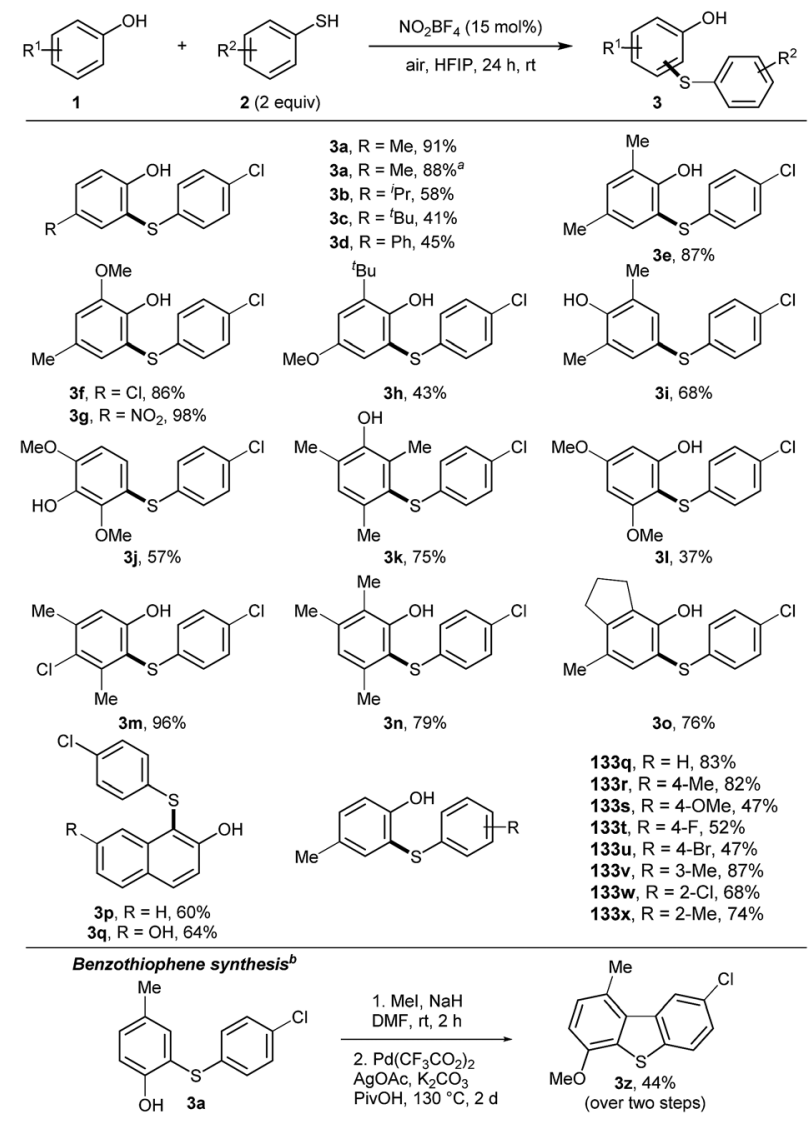

Scheme 1 Scope with respect to phenols (1) and thiophenols (2). Reaction conditions: 1 ( $0.1 \mathrm{mmol}, 1$ equiv.), 2 (2 equiv.), HFIP (0.05 M), at room temperature under air atmosphere. Yields are given for isolated products after column chromatography. ${ }^{a}$ Reaction carried out with $1 \mathrm{mmol}$ of phenol 1a. ${ }^{b}$ 1. 3a ( $0.3 \mathrm{mmol}, 1$ equiv.), Mel (1.5 equiv.), $\mathrm{NaH}$ (1.2 equiv.) in DMF $(0.1 \mathrm{M})$ at room temperature; 2. $\left(\mathrm{CF}_{3} \mathrm{CO}_{2}\right)_{2} \mathrm{Pd}(20 \mathrm{~mol} \%), \mathrm{AgOAC}$ (5 equiv.), $\mathrm{K}_{2} \mathrm{CO}_{3}$ (1.5 equiv.) in $\mathrm{PivOH}(0.3 \mathrm{M})$ at $130{ }^{\circ} \mathrm{C}$ for $2 \mathrm{~d}$.

Initially, the scope for the cross-coupling of phenols and thiophenols was studied (Scheme 1). The reaction was scaled to $1 \mathrm{mmol}$, which did not alter the outcome of the reaction. Functional groups at the para-position of phenols were well tolerated (3b-3d). 2,4-Substituted phenols yielded products $\mathbf{3 e}-\mathbf{3 h}$ in good to excellent yields, covering electron-rich and sterically demanding functional groups. Product $\mathbf{3 g}$ was isolated in quantitative yield and product $\mathbf{3 1}$ was synthesized with high paraselectivity. Electron-rich product $3 \mathbf{j}$ revealed selectivity for the meta-position of phenol. The same outcome was observed for product 3k by blocking the ortho- and para-positions. Dearomatization and subsequent 1,4-addition appeared to be an alternative pathway. $\mathbf{3} \mathbf{l}$ was isolated in moderate yield, using a 3,5-subsituted phenol. Polysubstituted phenols allowed the isolation of products 3m-o in 76-98\% yields. Naphthol derivatives were compatible, affording products 3p, 3q. Next, substituted thiophenols were tested. Different functional groups on the para-position were well tolerated $(3 \mathbf{s}-\mathbf{v})$. Alkyl or chloro substituents at the orthoand meta-position afforded products $3 \mathbf{w}-\mathbf{3 y}$ in good yields. Double thioarylation was not observed under the developed conditions. Alkyl and benzyl thiols did not yield the desired products either. To stress the utility of the obtained products, 3a was transformed into benzothiophene $\mathbf{3 z}$ by applying a dual $\mathrm{C}-\mathrm{H}$ bond activation strategy. ${ }^{17}$

Next, the thioarylation of indoles was studied (Scheme 2). Unprotected indoles gave better results than $N$-protected analogues. This result makes the reaction conditions more attractive for other applications. The cross-coupling of indole 4a and thiophenol 2a yielded $\mathbf{5 a}$ in $77 \%$ yield. Scaling the reaction to $1 \mathrm{mmol}$ gave 5a unaffectedly. Functional groups with different electronic properties at the indole skeleton were well tolerated (5b-f). Further, thiophenols were decorated with functional groups at the para $(\mathbf{5 h}-\mathbf{j})$, ortho $(\mathbf{5 k} \mathbf{k} \mathbf{- 1})$ and meta $(\mathbf{5 m})$ position. Product $5 \mathrm{n}$ was isolated in $51 \%$ yield bearing a napthyl moiety. Polysubstituted products $50-5$ s were synthesized in good yields, covering combinations of electron-rich and electron-deficient functional groups. Next, substituted pyrroles were tested. Product 5t was isolated in $64 \%$ yield. 2-Substituted pyrrol yielded the double functionalized product $\mathbf{5 v}$ in good yield. To further stress the applicability, $\mathbf{5 l}$ was transformed into the indol-fused benzothiophenes $\mathbf{5 v}{ }^{18}$

Next, the time course of the cross-coupling reactions was analysed by GC-MS-FID (Fig. 2). Interestingly, thiophenol 2a was fully converted to disulfide $\mathbf{6 a}$, prior to the coupling step with phenol 1a (Fig. 2A). In contrast, indole 4a and thiophenol 2a underwent synchronous cross-coupling without initial

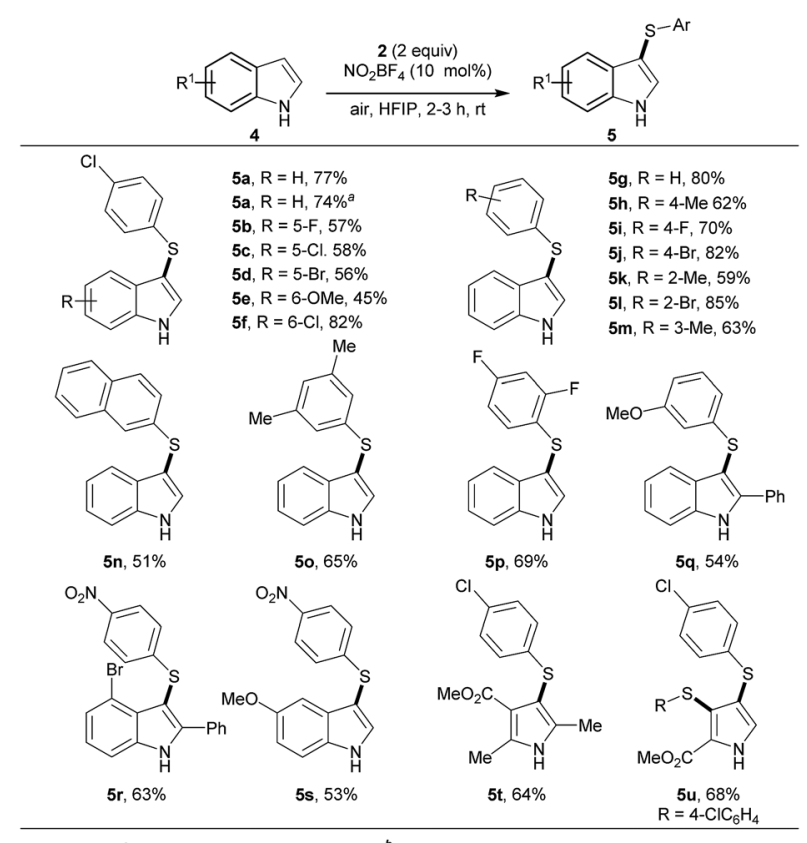

Indole-fused benzothiophene synthesist

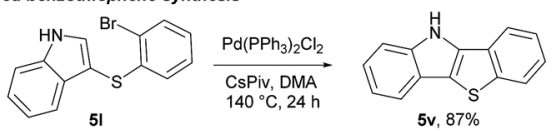

Scheme 2 Scope with respect to indoles (4) and thiophenols (2). Reaction conditions: 4 ( $0.1 \mathrm{mmol}, 1$ equiv.), 2 (2 equiv.), HFIP (0.05 M), at room temperature under air atmosphere. Yields are given for isolated products after column chromatography. ${ }^{a}$ Reaction carried out with $1 \mathrm{mmol}$ of indol 4 a. ${ }^{b} \mathbf{5 l}\left(0.08 \mathrm{mmol}, 1\right.$ equiv.), $\mathrm{Pd}\left(\mathrm{PPh}_{3}\right)_{2} \mathrm{Cl}_{2}$ (5 mol\%), CsPiv (2 equiv.) in $\mathrm{N}, \mathrm{N}$-dimethylacetamide (0.1 M). 

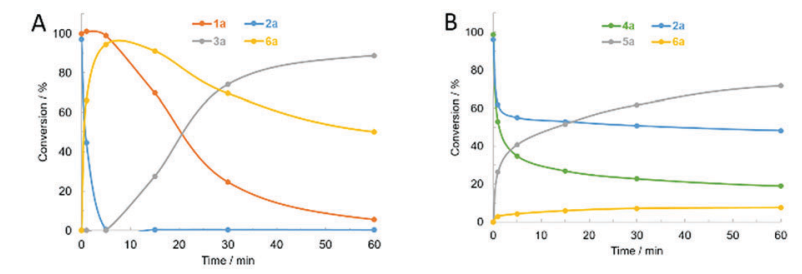

C
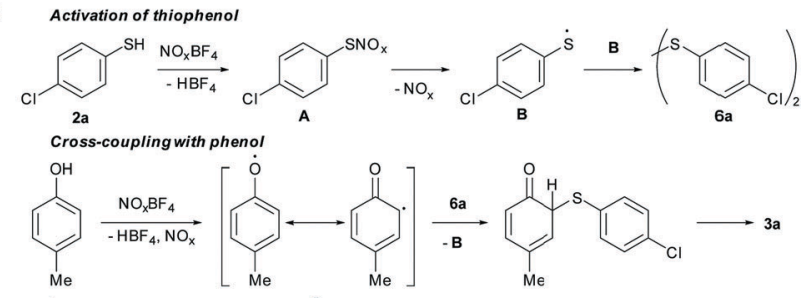

Cross-coupling with indole

$\underset{\mathrm{H}}{\stackrel{\mathrm{NO}_{x} \mathrm{BF}_{4}}{-\mathrm{BF}_{4} ; \mathrm{NO}_{x}}}$

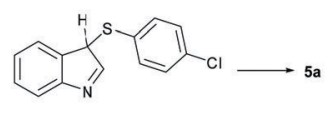

Regeneration of $\mathrm{NO}_{\mathrm{x}}^{+}$

$2 \mathrm{NO}_{2} \mathrm{BF}_{4} \frac{2 \mathrm{e}, 2 \mathrm{H}^{+}}{-2 \mathrm{HBF}_{4}} 2 \mathrm{NO}_{2} \longrightarrow \mathrm{N}_{2} \mathrm{O}_{4} \longrightarrow \mathrm{NO}^{+} \mathrm{NO}_{3} \frac{2 \mathrm{HBF}_{4}}{-\mathrm{H}_{2} \mathrm{O}} \mathrm{NOBF}_{4}+\mathrm{NO}_{2} \mathrm{BF}_{4}$
$\mathrm{NOBF}_{4} \stackrel{\mathrm{e}, \mathrm{H}^{+}}{\longrightarrow} \mathrm{NO} \stackrel{1 / 2 \mathrm{O}_{2}}{\longrightarrow} \mathrm{NO}_{2}$

Fig. 2 Reaction profiles and proposed mechanism. (A) Reaction profiles for the thioarylation of phenol $1 \mathbf{a}$ with thiophenol 2a. (B) Reaction profiles for the thioarylation of indole $\mathbf{4 a}$ with thiophenol $\mathbf{2 a}$. (C) Reaction mechanism.

formation of $\mathbf{6 a}$ (Fig. 2B). Conducting the coupling reaction with disulfide 6a confirmed the discrete recombination selectivities (Schemes S2 and S5, ESI $\dagger$ ). Consequently, disulfide 6a is an intermediate for the coupling with phenol, but indoles undergo direct recombination with thiophenols. Further control experiments were conducted (ESI $\dagger$ for the details). No product was formed in the presence of radical trap butylated hydroxytoluene (BHT) and the product formation was inhibited under inert gas atmosphere (Schemes S2 and S4, ESI $\dagger$ ). Ambient oxygen was crucial to maintain the catalytic activity. Initiation of the coupling reaction through oxidation of weak heteroatomhydrogen bonds was studied by methylating the starting materials. Drastically reduced conversion was observed, which excludes a direct single-electron-transfer (Schemes S2 and S4, ESI $\dagger$ ). Thiophenol 2a was quantitatively oxidized to disulfide $\mathbf{6 a}$ in the presence of $\mathrm{NOBF}_{4}$ (Scheme S3, ESI $\dagger$ ). Conversion to disulfide $6 \mathbf{6}$ was tied to the presence of air and did not take place in the presence of BHT (Scheme S3, ESI $\dagger$ ).

Based on the control experiments, a mechanism for the cross-coupling of phenol 1a and indole 4a with thiophenol 2a was proposed (Fig. 2C). Initially, intermediate $\mathbf{A}$ is formed by $S$-nitrosylation of $\mathbf{2 a}$. Homolytic cleavage releases radical $\mathbf{B}$, which forms disulfide 6a by recombination with itself. Phenol 1a is oxidized to form a phenoxy radical. Delocalization of the phenoxy radical (C) and attack at the disulfide bond leads to release of radical $\mathbf{B}$. Rearomatization furnishes product $\mathbf{3 a}$. Oxidation of indole $\mathbf{4 a}$ gives intermediate $\mathbf{D}$, which undergoes recombination with $\mathbf{B}$. Oxidation of indole $\mathbf{4 a}$ and thiophenol 2a occurs synchronously, leading to the formation of $\mathbf{5 a}$ before disulfide 6a begins to form. Further, $\mathrm{NO}_{2} \mathrm{BF}_{4}$ oxidizes the starting materials to form $\mathrm{NO}_{2}$. $\mathrm{NO}_{2}$ dimerizes to form $\mathrm{N}_{2} \mathrm{O}_{4}$, which undergoes disproportionation to $\mathrm{NONO}_{3}{ }^{11 a}$ Water is released upon protonation by $\mathrm{HBF}_{4}$ and nitrosonium and nitronium tetrafluoroborate are regenerated. $\mathrm{NOBF}_{4}$ is capable of oxidizing the substrates in the same way as the nitronium salt. Oxidation of substrates results in the formation of nitrogen monoxide, which has to be oxidized by ambient oxygen, in order to maintain the catalytic cycle.

Based on the revealed radical mechanism, we hypothesized that phenothiazines represent suitable substrates for the radical recombination with phenols, due to the low N-H BDE and the unique ability to stabilize radical intermediates. ${ }^{19}$ Multi parameter optimization was performed (Table S2, ESI $\dagger$ ). Sustainable $\mathrm{C}-\mathrm{H}$ bond amination of phenols with phenothiazines was achieved using $\mathrm{NaNO}_{2}$ as catalyst, omitting halogenated reagents and solvents. The developed conditions overcome environmental issues of known methods. ${ }^{7}$

Next, the scope of the catalytic C-H/N-H cross-dehydrogenative coupling was studied (Scheme 3). Initially, the cross-coupling of 4-methoxyphenol (7a) and different phenothiazines was performed. Products 9a-d were isolated in good yields. Different 4-substituted phenols yielded the desired cross-coupling products in moderate to excellent yields (9e-j). Cross-coupling with differently substituted phenothiazines was achieved for several phenols. Product $9 \mathbf{k}$ was isolated in good yield as single regioisomer. Polyfunctionalized products $\mathbf{9 l - q}$ were isolated in moderate to good yields. Phenoxazine proved to be compatible

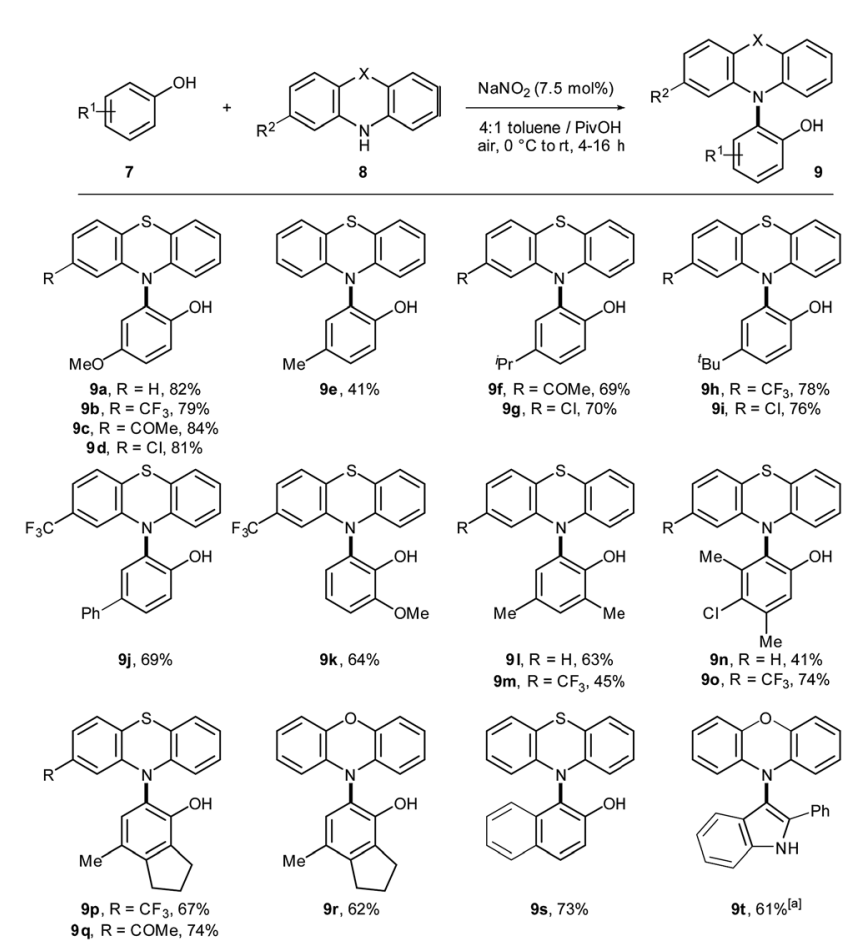

Scheme 3 Scope for the cross-dehydrogenative $\mathrm{C}-\mathrm{H}$ bond amination. Reaction conditions: 8 ( $0.2 \mathrm{mmol}, 1$ equiv.), 9 (3 equiv.), $4: 1$ toluene/ $\mathrm{PivOH}$ $(0.05 \mathrm{M}), 0{ }^{\circ} \mathrm{C}$ to $\mathrm{rt}$ under air atmosphere. Yields are given for isolated products after column chromatography. ${ }^{a} \mathrm{NOBF}_{4}(0.02 \mathrm{mmol}, 10 \mathrm{~mol} \%)$ was used as catalyst. 
for the cross-coupling reaction (9r). 2-Naphthol yielded the desired product $9 \mathrm{~s}$ in $73 \%$ yield. Finally, the cross-coupling of 2-phenylindole and phenoxazine was successfully performed. However, synthesis of $9 \mathbf{t}$ worked superior if $\mathrm{NOBF}_{4}$ was used as catalyst. The underlying mechanism for the aerobic $\mathrm{C}-\mathrm{H}$ bond amination proceeds analogously as described before via direct radical-radical recombination under aerobic conditions (Scheme S8, ESI $\dagger$ ).

In summary, we have reported the first application of $\mathrm{NO}_{x}^{+}$ as efficient and environmentally friendly catalyst for carbonheteroatom bond formation. The operationally simple and sustainable protocol enables the $\mathrm{C}-\mathrm{H} / \mathrm{S}-\mathrm{H}$ and $\mathrm{C}-\mathrm{H} / \mathrm{N}-\mathrm{H}$ CDC. Ambient oxygen serves as stoichiometric oxidant and water is generated as by-product. A broad scope was demonstrated in good yields and regioselectivities. The reported methodology offers mild reaction conditions and does not require an excess of reagents or any specialized equipment.

A. P. A. acknowledges the support of the DFG (AN 1064/4-1) and the Boehringer Ingelheim Foundation (Plus 3). L. B. is supported by the Verband der Chemischen Industrie e.V. We gratefully acknowledge Ms R. Perinbarajah for assistance. Open Access funding provided by the Max Planck Society.

\section{Conflicts of interest}

There are no conflicts to declare.

\section{Notes and references}

1 (a) J. F. Hartwig, Nature, 2008, 455, 314; (b) A. K. Yudin and J. F. Hartwig, Catalyzed Carbon-Heteroatom Bond Formation, Wiley-VCH, Weinheim, 2010.

2 (a) S. A. Girard, T. Knauber and C.-J. Li, Angew. Chem., Int. Ed., 2014, 53, 74; (b) J. F. Hartwig and M. A. Larsen, ACS Cent. Sci., 2016, 2, 281.

3 (a) I. P. Beletskaya and V. P. Ananikov, Chem. Rev., 2011, 111, 1596; (b) J. Bariwal and E. Van der Eycken, Chem. Soc. Rev., 2013, 42, 9283; (c) L. Chin-Fa, L. Yi-Chen and B. S. Singh, Chem. - Asian J., 2014, 9, 706; (d) M.-L. Louillat and F. W. Patureau, Chem. Soc. Rev., 2014, 43, 901; (e) Y. Park, Y. Kim and S. Chang, Chem. Rev., 2017, 117, 9247.

4 (a) C.-L. Sun and Z.-J. Shi, Chem. Rev., 2014, 114, 9219; (b) R. A. D. Arancon, C. Sze Ki Lin, C. Vargas and R. Luque, Org. Biomol. Chem., 2014, 12, 10; (c) V. P. Mehta and B. Punji, RSC Adv., 2013, 3, 11957.

5 (a) R. Samanta, K. Matcha and A. P. Antonchick, Eur. J. Org. Chem., 2013, 5769; (b) R. Narayan, K. Matcha and A. P. Antonchick, Chem. - Eur. J., 2015, 21, 14678; (c) L. Bering, S. Manna and A. P. Antonchick, Chem. - Eur. J., 2017, 23, 10936.

6 (a) Y. Liu, Y. Zhang, C. Hu, J.-P. Wan and C. Wen, RSC Adv., 2014, 4, 35528; (b) S. K. R. Parumala and R. K. Peddinti, Green Chem., 2015, 17, 4068; (c) Z. Huang, D. Zhang, X. Qi, Z. Yan, M. Wang, H. Yan and A. Lei, Org. Lett., 2016, 18, 2351; (d) S. Song, Y. Zhang, A. Yeerlan, B. Zhu, J. Liu and N. Jiao, Angew. Chem., Int. Ed., 2017, 56, 2487; (e) L. T. Silva, J. B. Azeredo, S. Saba, J. Rafique,
A. J. Bortoluzzi and A. L. Braga, Eur. J. Org. Chem., 2017, 4740; $(f)$ R. Rahaman, S. Das and P. Barman, Green Chem., 2018, 20, 141; (g) H.-H. Wang, T. Shi, W.-W. Gao, Y.-Q. Wang, J.-F. Li, Y. Jiang, Y. S. Hou, C. Chen, X. Peng and Z. Wang, Chem. - Asian J., 2017, 12, 2675; (h) P. Wang, S. Tang, P. Huang and A. Lei, Angew. Chem., Int. Ed., 2017, 56, 3009; (i) R. Ohkado, T. Ishikawa and H. Iida, Green Chem., 2018, 20, 984; $(j)$ H. Iida, R. Demizu and R. Ohkado, J. Org. Chem., 2018, 83, 12291.

7 (a) M.-L. Louillat-Habermeyer, R. Jin and F. W. Patureau, Angew. Chem., Int. Ed., 2015, 54, 4102; (b) R. Jin and F. W. Patureau, Org. Lett., 2016, 18, 4491; (c) Y. Zhao, B. Huang, C. Yang and W. Xia, Org. Lett., 2016, 18, 3326; (d) M. Goswami, A. Konkel, M. Rahimi, M.-L. Louillat-Habermeyer, H. Kelm, R. Jin, B. de Bruin and F. W. Patureau, Chem. - Eur. J., 2018, 24, 11936; (e) R. Jin, C. L. Bub and F. W. Patureau, Org. Lett., 2018, 20, 2884; $(f)$ S. Tang, S. Wang, Y. Liu, H. Cong and A. Lei, Angew. Chem., Int. Ed., 2018, 57, 4737.

8 (a) K. Y. Lee, D. J. Kuchynka and J. K. Kochi, Inorg. Chem., 1990, 29, 4196; (b) G. A. Olah, G. K. S. Prakash, Q. Wang and X.-Y. Li, Nitrosonium Tetrafluoroborate, Encyclopedia of Reagents for Organic Synthesis, John Wiley \& Sons, New Jersey, 2001; (c) G. I. Borodkin and V. G. Shubin, Russ. Chem. Rev., 2001, 70, 211; (d) I. B. Gennady and G. S. Vyacheslav, Russ. Chem. Rev., 2017, 86, 18.

9 F. Radner, J. Org. Chem., 1988, 53, 702.

10 (a) M. Tanaka, H. Nakashima, M. Fujiwara, H. Ando and Y. Souma, J. Org. Chem., 1996, 61, 788; (b) M. Tanaka, E. Muro, H. Ando, Q. Xu, M. Fujiwara, Y. Souma and Y. Yamaguchi, J. Org. Chem., 2000, 65, 2972.

11 (a) B. Su, L. Li, Y. Hu, Y. Liu and Q. Wang, Adv. Synth. Catal., 2012, 354, 383; (b) B. Su, M. Deng and Q. Wang, Org. Lett., 2013, 15, 1606.

12 (a) Y. E. Lee, T. Cao, C. Torruellas and M. C. Kozlowski, J. Am. Chem. Soc., 2014, 136, 6782; (b) A. Libman, H. Shalit, Y. Vainer, S. Narute, S. Kozuch and D. Pappo, J. Am. Chem. Soc., 2015, 137, 11453; (c) M. Koji, S. Kazuma, O. Takao, D. Toshifumi and K. Yasuyuki, Angew. Chem., Int. Ed., 2016, 55, 3652; (d) H. Shalit, A. Libman and D. Pappo, J. Am. Chem. Soc., 2017, 139, 13404; (e) A. Wiebe, B. Riehl, S. Lips, R. Franke and S. R. Waldvogel, Sci. Adv., 2017, 3, eaao3920; $(f)$ H. Kang, Y. E. Lee, P. V. G. Reddy, S. Dey, S. E. Allen, K. A. Niederer, P. Sung, K. Hewitt, C. Torruellas, M. R. Herling and M. C. Kozlowski, Org. Lett., 2017, 19, 5505; (g) S. R. Waldvogel, S. Lips, M. Selt, B. Riehl and C. J. Kampf, Chem. Rev., 2018, 118, 6706.

13 (a) L. Bering, F. M. Paulussen and A. P. Antonchick, Org. Lett., 2018, 20, 1978; (b) L. Bering, M. Vogt, F. M. Paulussen and A. P. Antonchick, Org. Lett., 2018, 20, 4077.

14 (a) T. Itoh, N. Tsutsumi and A. Ohsawa, Bioorg. Med. Chem. Lett., 1999, 9, 2161; (b) A. S. Demir, I. A. Cigdem and A. S. Mahasneh, Tetrahedron, 1999, 55, 12399; (c) N. Tsutsumi, T. Itoh and A. Ohsawa, Chem. Pharm. Bull., 2000, 48, 1524; (d) J.-M. Lü, J. M. Wittbrodt, K. Wang, Z. Wen, H. B. Schlegel, P. G. Wang and J.-P. Cheng, J. Am. Chem. Soc., 2001, 123, 2903.

15 (a) M. I. de Heer, H.-G. Korth and P. Mulder, J. Org. Chem., 1999, 64, 6969; (b) Y.-D. Wu and D. K. W. Lai, J. Org. Chem., 1996, 61, 7904.

16 (a) L. Eberson, M. P. Hartshorn and O. Persson, J. Chem. Soc., Perkin Trans. 2, 1995, 1735, DOI: 10.1039/P29950001735; (b) M. Lucarini, V. Mugnaini, G. F. Pedulli and M. Guerra, J. Am. Chem. Soc., 2003, 125, 8318; (c) J.-P. Bégué, D. Bonnet-Delpon and B. Crousse, Synlett, $2004,18$.

17 C. Rui, W. Zhiqing, L. Zhengkai, X. Haifeng and Z. Xiangge, Chem. - Eur. J., 2014, 20, 7258.

18 P. Saravanan and P. Anbarasan, Org. Lett., 2014, 16, 848.

19 (a) M. Lucarini, P. Pedrielli, G. F. Pedulli, L. Valgimigli, D. Gigmes and P. Tordo, J. Am. Chem. Soc., 1999, 121, 11546; (b) L. A. Farmer, E. A. Haidasz, M. Griesser and D. A. Pratt, J. Org. Chem., 2017, 82, 10523. 